\title{
Pharmaceutical Care in a Patient with Recurrent Infection after Liver Transplant
}

\author{
Liqin Zhu ${ }^{1}$ and Xiaoye Sun ${ }^{2}$ \\ ${ }^{1}$ Department of Pharmacy, Tianjin First Central Hospital, Tianjin, China \\ ${ }^{2}$ Department of Organ Transplant, Tianjin First Central Hospital, Tianjin, China
}

\begin{abstract}
Case (description): A case of a 57-year-old liver transplant patient with biliary drainage catheter whose recurrent infection was managed by clinical pharmacists. The suggestions about the choice of antibiotic regimen and the monitoring to other related infection factors, such as biliary drainage catheter and concentration of tracrolimus, were accepted and used to the patient. The state of the infected patient was improved.

Conclusion: The antibiotics' distribution, antibacterial effect, and drug resistance could be taken into consideration for the patients with recurrent infection after liver transplant. The resistance of antibiotics could be deferred by altering suitable antibiotics. Close care to catheter and immunosuppressants could be necessary to prevent infection for the patients after transplant.
\end{abstract}

Keywords: Pharmaceutical care; Recurrent infection; Liver transplant

\section{Introduction}

The recurrences of bacteria and virus infection are common problems in liver transplant recipients because of the biliary drainage catheter and their extended state of immunosuppression. In recent years, gram-negative rods have emerged as the predominant pathogens in solid organ transplant (SOT) recipients [1-3]. Among these gramnegative rods, Klebsiella pneumonia (K. pneumoniae) was the most common bacteria identified in some case studies [3], and SOT has been implicated as a factor predisposing patients to K. pneumoniae bloodstream infection (BSI) [4]. Carbapenems, which are regarded as effective agents for treating K. pneumonia infection, induce the production and global spread of Klebsiella pneumoniae carbapenemase (KPC) [5]. Although polymyxin B combined with tigecycline could be used for carbapenem-resistant K. pneumoniae (CRKP) [6], limitations to the application of carbapenems are of great concern in K. pneumonia therapy.

This case report provided a series of therapeutic strategies, from view of the clinical pharmacist, in a patient with recurrent $K$. pneumoniae infection after liver transplant.

Ethical approval: No approval was necessary

\section{Case Report}

The patient was a 57-year-old man diagnosed with Hepatitis B virus (HBV)-related cirrhosis and type II diabetes. He underwent liver transplant surgery in December 2009. After his surgery, ursodeoxycholic acid and losartan potassium/hydrochlorothiazide were administered discontinuously and biliary drainage catheter was kept in place because of elevated bilirubin levels and blood pressure. Tacrolimus (FK506) was administered at a decreasing dose and its concentration was within the therapeutic window (Figure 1). He was examined by his physician at the beginning of 2014 because of his recurrent infection in the third year after surgery. The patient was tested for K. pneumoniae and Enterococcus faecium 40 and 35 times, respectively, from December 2012 to March 2014. Most test results were normal. The patient suffered infections for three times (one in December 2012 and two in January 2013) induced by K. pneumoniae. His white blood cell (WBC) count, total bile acid (TBIL), alanine aminotransferase (ALT) and aspartate aminotransferase (AST) varied during this period, and there was no notable increase except for neutrophils (Neut) (Figure 2-6). WBC and Neut were fluctuated. Neut were higher than normal for three times. Although there were only three times abnormal blood chemistry data, he was still administered meropenem intravenously when abnormal bile occurred or when test results were positive for bacteria in his bile. His blood sugar was well controlled in this period. The patient consulted his doctor whether this anti-bacterial dosage regimen was suitable.

Considering the details of the patient and the general condition (pharmacokinetics, efficacy, and drug resistance) of antibiotics, clinical pharmacists provided the following suggestions. These suggestions were accepted by the doctor. By applying the series of therapeutic strategies to the patient, the state of the infected patient was improved.

1. Ensuring that the biliary drainage catheter was still in need. If needed, careful care of the catheter was required, such as inspecting and flushing the catheter twice daily, and emptying collected drainage from the bag daily.

2. Carbapenems, cephalosporins, and broad-spectrum penicillin antibiotics could be used in turn after confirming the infection in the patient.

3. Monitoring tacrolimus concentrations to avoid low immunity which could be result in infection.

\section{Discussion}

In special population of liver transplants, bacteria in bile were more frequent. It was important that determining the relationship between bacteria in bile and infection. Besides the antimicrobial spectrum of antibiotics, pharmacokinetic parameters, especially the distribution

*Corresponding author: Liqin Zhu, Pharm D, Chinical Pharmacist, Pharmacy Dept., Tianjin First Center Hospital, Tianjin, China, No.24 Fukang Road, Nankai District, 300192, China, Tel: +86 022 23626417; E-mail: zlq0713@aliyun.com

Received November 04, 2014; Accepted November 28, 2014; Published December 01, 2014

Citation: Zhu L, Sun X (2014) Pharmaceutical Care in a Patient with Recurrent Infection after Liver Transplant. Clin Exp Pharmacol 4: 166. doi:10.4172/21611459.1000166

Copyright: $\odot 2014$ Zhu L, et al. This is an open-access article distributed under the terms of the Creative Commons Attribution License, which permits unrestricted use, distribution, and reproduction in any medium, provided the original author and source are credited. 
Citation: Zhu L, Sun X (2014) Pharmaceutical Care in a Patient with Recurrent Infection after Liver Transplant. Clin Exp Pharmacol 4: 166. doi:10.4172/2161-1459.1000166

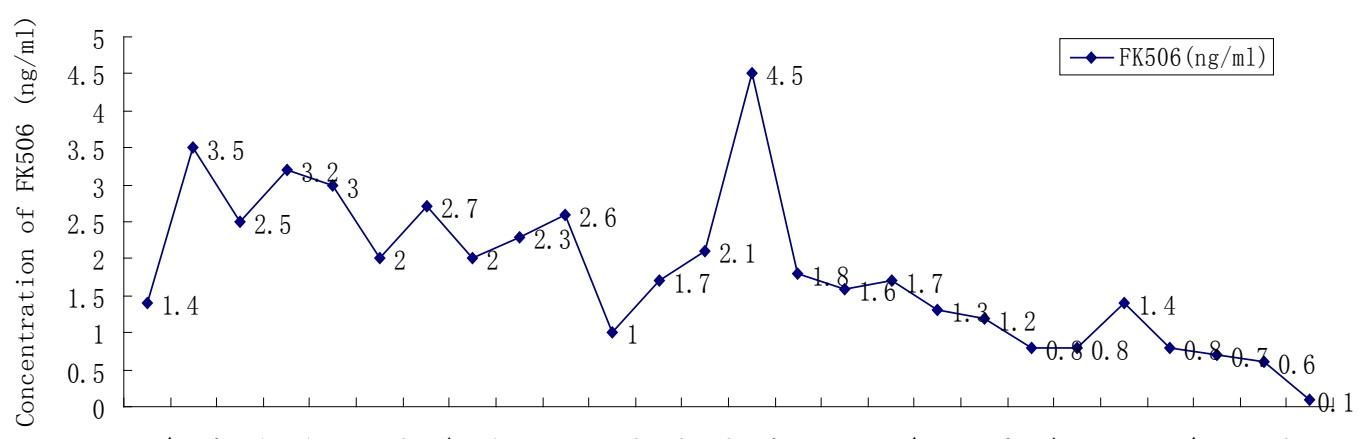

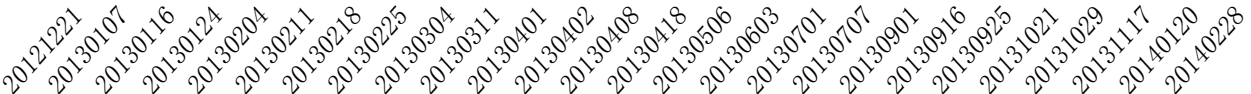

Figure 1: Laboratory data of tacrolimus in the patient.

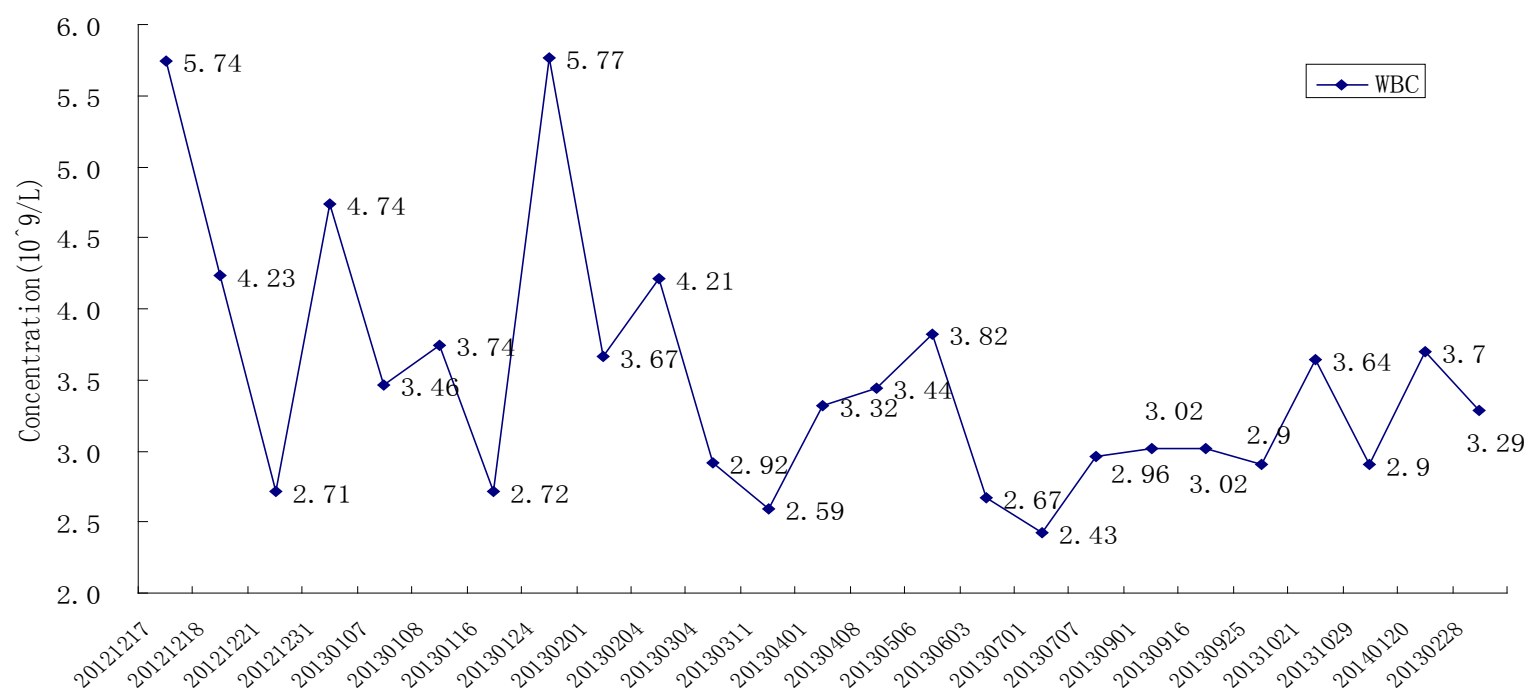

Figure 2: Laboratory data of WBC in the patient.

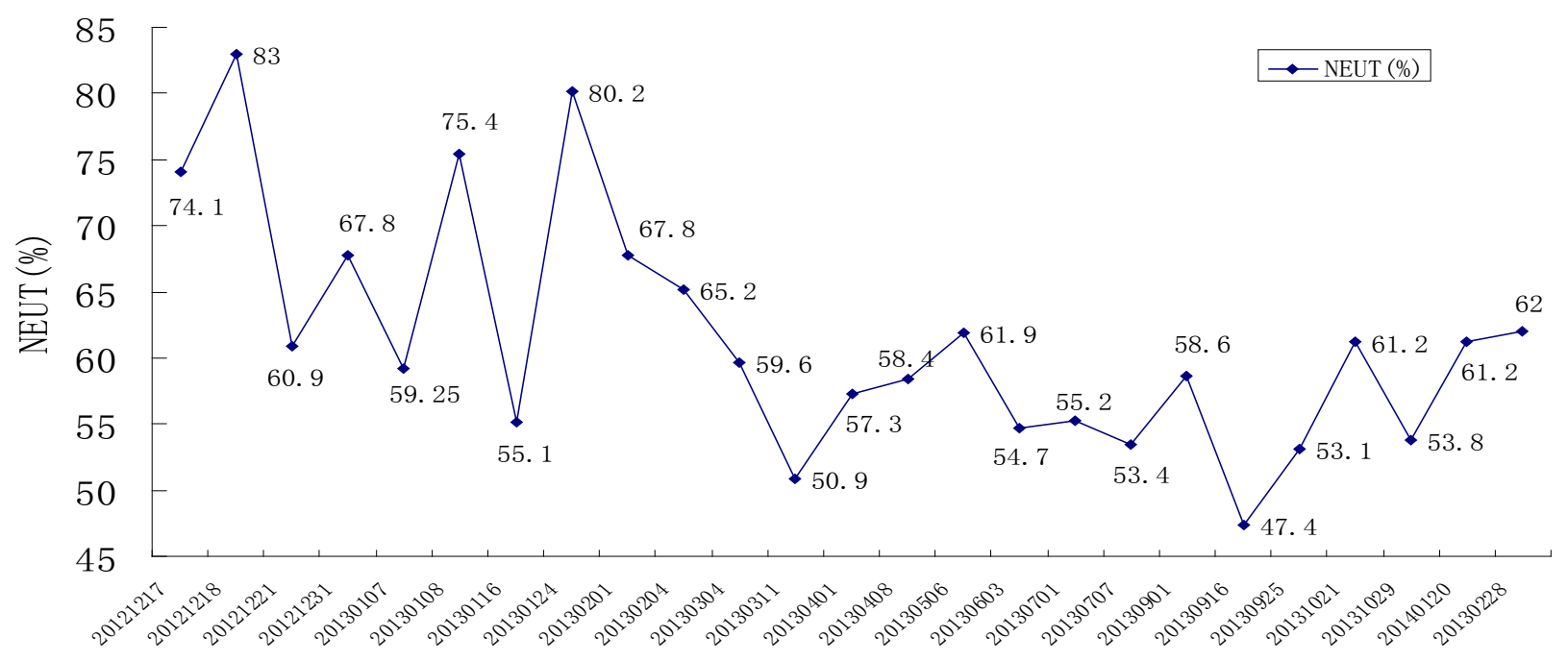

Figure 3: Laboratory data of Neut in the patient. 
Citation: Zhu L, Sun X (2014) Pharmaceutical Care in a Patient with Recurrent Infection after Liver Transplant. Clin Exp Pharmacol 4: 166. doi:10.4172/2161-1459.1000166
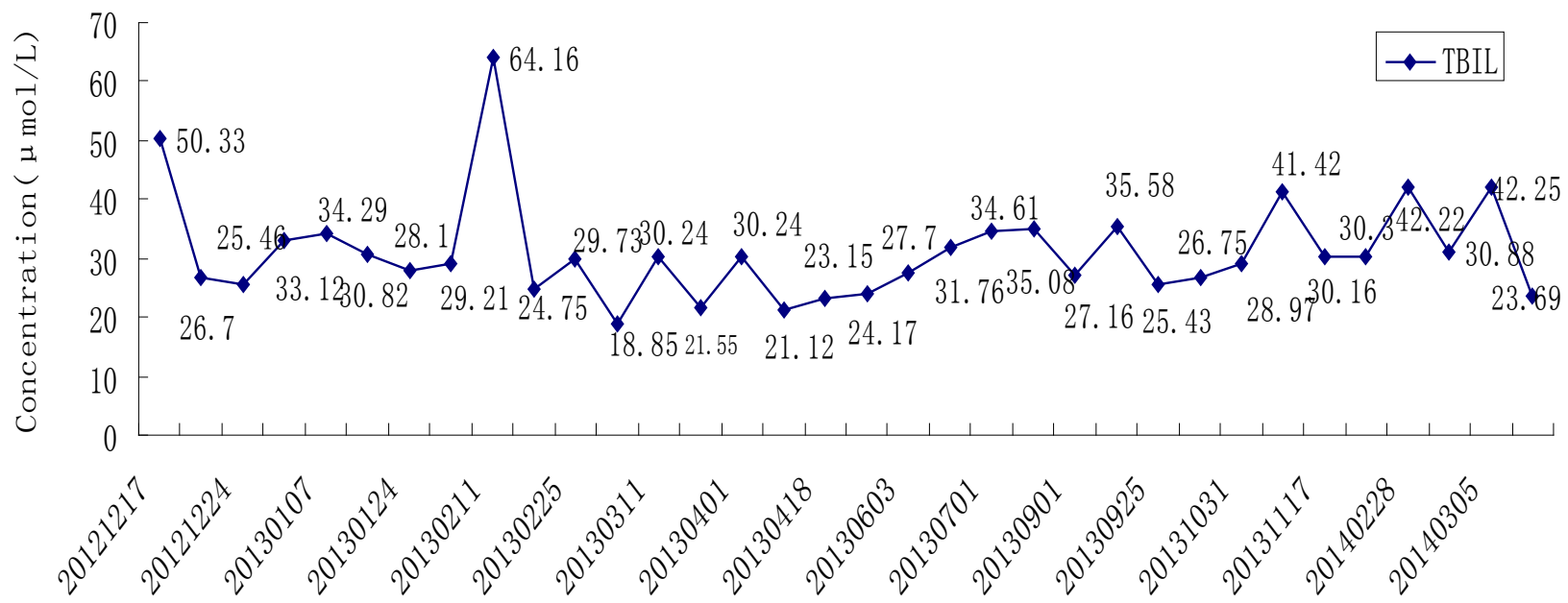

Figure 4: Laboratory data of TBIL in the patient.

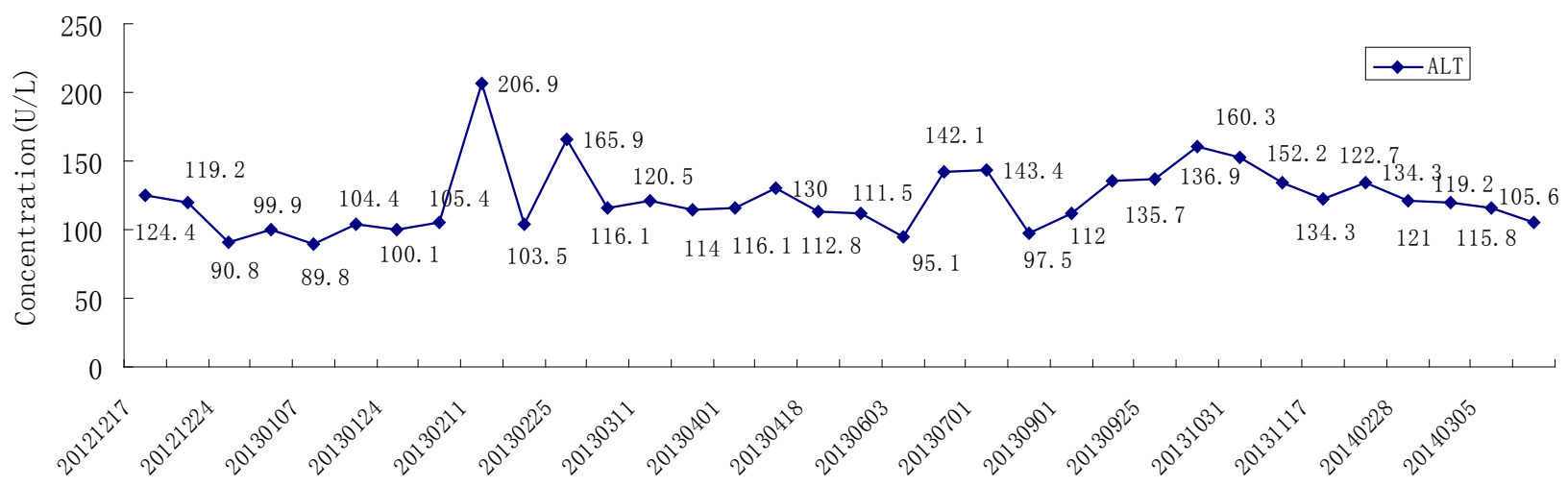

Figure 5: Laboratory data of ALT in the patient.

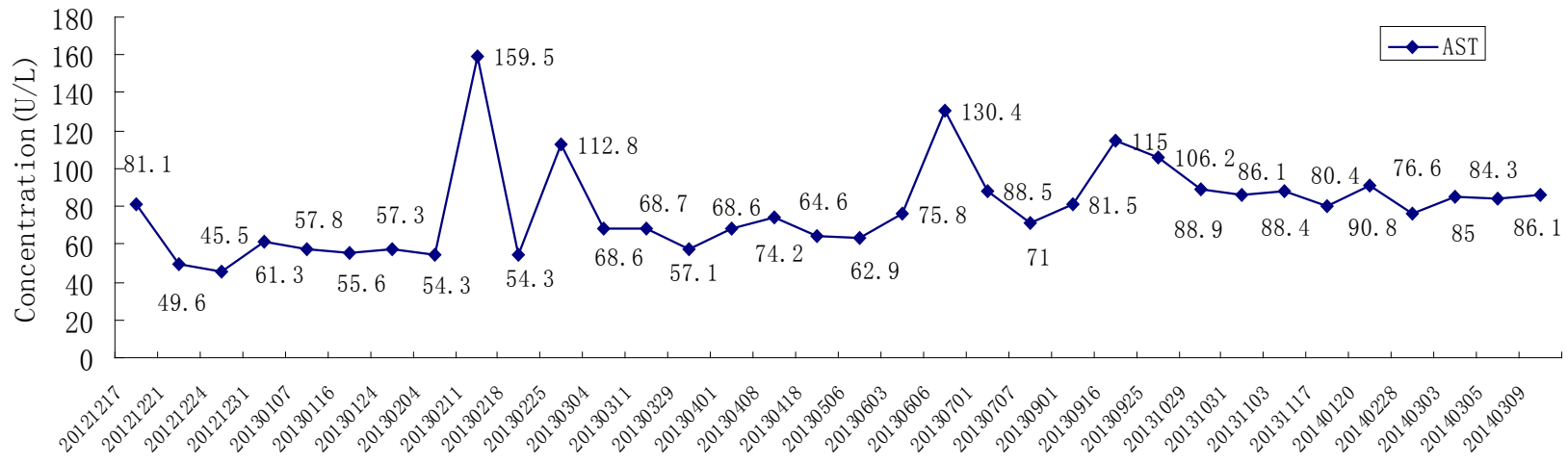

Figure 6: Laboratory data of AST in the patient.

of antibiotics, should be considered while choosing antibiotics for the patients with recurrent infection.

K. pneumoniae [7] and Enterococcus faecium [8] were common bacteria in the human intestinal tract. Only under certain conditions could infections be induced. For this patient, the liver transplant surgery and extended use of immunosuppressants after surgery, which maintained reduced immunity, made infection easier. Moreover, the biliary drainage catheter and diabetes also increase the incidence of infection.

While infection occurred after liver transplant, antibiotic use should be considered for this patient. Carbapenems such as meropenem were usually used in patients after liver transplant because of their higher 
Citation: Zhu L, Sun X (2014) Pharmaceutical Care in a Patient with Recurrent Infection after Liver Transplant. Clin Exp Pharmacol 4: 166. doi:10.4172/2161-1459.1000166

\begin{tabular}{|c|c|c|c|}
\hline & $\begin{array}{c}\text { Hepatobiliary } \\
\text { System } \\
\text { Distribution }\end{array}$ & $\begin{array}{c}\text { Anti-infection } \\
\text { Effect }\end{array}$ & $\begin{array}{c}\text { Drug } \\
\text { Resistance }\end{array}$ \\
\hline Fluoroquinolones & - & + & ++ \\
\hline Carbopenems & ++ & +++ & ++ \\
\hline Cephalosporins & +++ & ++ & + \\
\hline Broad-spectrum Penicillins & +++ & ++ & + \\
\hline
\end{tabular}

Note:,,++++++ : the distribution and drug resistance varied from low $(+)$ to high $(+++)$; the anti-infection effect varied from weak $(+)$ to strong $(+++)$; -: no distribution

Table 1: Comparison between different kinds of antibiotics.

anti-bacterial effectiveness. The patient was prescribed meropenem as soon as the bacteria were detected. However, the bacterial test results did not accurately represent the infection. The physician had to ensure that the antibiotic use was needed to the patient. Moreover, clinical pharmacist suggested that the pharmacokinetics, pharmacodynamics and drug resistance conditions should be taken into consideration. Detailed information was presented in Table 1. Of all the anti-Klebsiella pneumoniae drugs, only fluoroquinolones (FQNs) have poor distribution in the hepatobiliary system. Therefore, FQNs should not be applied in this patient. Although the effectiveness of carbapenems against infection was higher than cephalosporins and broad-spectrum penicillins, their lower distribution in the hepatobiliary system and higher drug resistance somehow limited their using. Clinical pharmacists suggested alternating use among the three kinds of antibiotics to avoid resistance. For some antibiotics that were metabolized by liver (such as cefoperazone), the side effects, especially liver-related side effects and drug-drug interactions, should be carefully monitored by clinical pharmacists.

Additional information should be considered to prevent inflammation, as follows: 1) cleaning the biliary drainage catheter. The biliary drainage catheter should be flushed twice daily using normal saline and the bag should also be emptied daily. The dressing stopcock and the drainage bag should be changed once a week. The dressing should be changed more frequently if it became loose, wet, or soiled; and 2) monitoring immunosuppressant. An infection after liver transplant is often related to lower immunity. Immunosuppressant monitoring was required for the rest of his life after the transplant.
The therapeutic strategy for patients with recurrent K. pneumonia infection afterliver transplant should becarefullyconsidered. The catheter should be sterilized according to the protocol, immunosuppressant should be controlled and managed, and appropriate antibiotics should be chosen. Anti-K. pneumonia antibiotics should be rotated between carbapenems, cephalosporins, and broad-spectrum penicillins by taking the anti-infection effect, drug distribution, and drug resistance into consideration.

\section{Acknowledgment}

This work was supported by National High Technology Development Project (2012AA021003) and the key Projects of Tianjin Health Bureau(12KG103).

\section{References}

1. Linares L, Garcia-Goez JF, Cervera C, Almela M, Sanclemente G, et al. (2009) Early bacteremia after solid organ transplantation. Transplant Proc 41: 2262 2264 .

2. Al-Hasan MN, Razonable RR, Eckel-Passow JE, Baddour LM, (2009) Incidence rate and outcome of gram-negative bloodstream infection in solid organ transplant recipients. Am J Transplant 9: 835-843.

3. Singh N, Wagener MM, Obman A, Cacciarelli TV, de Vera ME, et al. (2004) Bacteremias in liver transplant recipients: shift toward gram-negative bacteria as predominant pathogens. Liver Transpl 10: 844-849.

4. Meatherall BL, Gregson D, Ross T, Pitout JD, Laupland (2009) KB Incidence, risk factors, and outcomes of Klebsiella pneumoniae bacteremia. Am J Med 122: 866-873

5. Bergamasco MD, Barroso Barbosa M, de Oliveira Garcia D, Cipullo R, Moreira JC et al. (2012) Infection with Klebsiella pneumoniae carbapenemase (KPC)producing K. pneumoniae in solid organ transplantation. Transpl Infect Dis 14: 198-205.

6. Lee J, Patel G, Huprikar S, Calfee DP, Jenkins SG (2009) Decreased Susceptibility to Polymyxin B during Treatment for Carbapenem-Resistant Klebsiella pneumoniae Infection. J Clin Microbiol 47:1611-1612.

7. Wong MT, Choi SB, Kuan CS, Chua SL, Chang CH, Normi YM, et al. (2012) Structural Modeling and Biochemical Characterization of Recombinant KPN 02809, a Zinc-Dependent Metalloprotease from Klebsiella pneumoniae MGH 78578. Int J Mol Sci 13: 901-917.

8. Castillo-Rojas G, Mazari-Hiríart M, Ponce de León S, Amieva-Fernández RI, Agis-Juárez RA, et al. (2013) Comparison of Enterococcus faecium and Enterococcus faecalis Strains Isolated from Water and Clinical Samples: Antimicrobial Susceptibility and Genetic Relationships. PLoS One 8:e59491. 